\title{
Comparison of ultrasound-guided stellate ganglion block at 6th and 7th cervical vertebrae using the lateral paracarotid out-of-plane approach for sympathetic blockade in the upper extremity
}

\author{
Jongyoon Baek', Bum Soo Kim², Hwarim $\mathrm{Yu}^{3}$, Hyuckgoo $\mathrm{Kim}^{4}$, Chaeseok $\mathrm{Lim}^{4}$, Sun Ok Song ${ }^{4}$ \\ ${ }^{I}$ Gajo-myeon Public Health Branch, Geochang; ${ }^{2}$ KIM BUM SOO PAIN CLINIC, Ulsan; \\ ${ }^{3}$ Department of Anesthesiology and Pain Medicine, Gangnam Severance Hospital, Seoul; \\ ${ }^{4}$ Department of Anesthesiology and Pain Medicine, Yeungnam University College of Medicine, Daegu, Korea
}

Background: The authors have performed ultrasound-guided stellate ganglion block (SGB) in our clinic using a lateral paracarotid approach at the level of the 6th cervical vertebra (C6). Although SGB at C6 is a convenient and safe method, there are ongoing concerns about the weak effect of sympathetic blockade in the ipsilateral upper extremity. Therefore, ultrasound-guided SGB was attempted using a lateral paracarotid approach at the level of the 7 th cervical vertebra (C7). This prospective study aimed to compare changes in skin temperature after SGB was performed at $\mathrm{C} 6$ and $\mathrm{C} 7$, and to introduce a lateral paracarotid approach for SGB.

Methods: Thirty patients underwent SGB twice: once at C6 and once at C7. For every SGB, the skin temperature of the patient's hypothenar area was measured for $15 \mathrm{~min}$ at 1-min intervals. Skin temperatures before and after SGB and side effects were compared between C6 and C7 groups.

Results: The temperature of the upper extremity increased after SGB was performed at $\mathrm{C} 6$ and $\mathrm{C} 7$. There were significant differences between mean pre-SGB and the largest increases in post-SGB temperatures $\left(0.50 \pm 0.38^{\circ} \mathrm{C}\right.$ and $1.41 \pm 0.68^{\circ} \mathrm{C}$ at $\mathrm{C} 6$ and $\mathrm{C} 7$, respectively; $\left.p<0.05\right)$. Significantly increased post-SGB temperatures (difference $>1{ }^{\circ} \mathrm{C}$ ) were found in $5 / 30(16.7 \%)$ and $24 / 30(80 \%)$ cases for $\mathrm{C} 6$ and $\mathrm{C} 7$, respectively $(p<$ $0.05)$. There were no significant differences in side effects between SGB performed at C6 or C7 ( $p>0.05)$. Conclusion: The lateral paracarotid approach using out-of-plane needle insertion for ultrasound-guided SGB performed at $\mathrm{C} 7$ was feasible and more effective at elevating skin temperature in the upper extremity than SGB at $\mathrm{C} 6$.

Keywords: Skin temperature; Stellate ganglion; Sympathetic nerve block; Ultrasonography; Upper extremity

\section{INTRODUCTION}

Stellate ganglion block (SGB) has been used since the mid-

Received: August 7, 2018, Revised: November 7, 2018 Accepted: November 9, 2018

Corresponding Author: Sun Ok Song, Department of Anesthesiology and Pain Medicine, Yeungnam University College of Medicine, 170, Hyeonchung-ro, Nam-gu, Daegu 42415, Korea

Tel: +82-53-620-3362, Fax: +82-53-626-5275

E-mail: sosong@ynu.ac.kr 1930s, and has become a common intervention for the treatment of sympathetically mediated pain and vascular insufficiency of the head, neck, and upper extremity. SGB has been performed at the level of the 6th cervical vertebra (C6), owing to the easy palpability of the $\mathrm{C} 6$ tubercle (Chassaignac's tubercle) in the neck, and the low risk for puncture of the vertebral artery and pneumothorax. In addition, the anterior paratracheal approach at the level of C6 can be performed blindly by many practitioners. Recent reports have suggested that ultrasound-guided SGB is a safer and more accurate me-

Copyright (C) 2018 Yeungnam University College of Medicine

This is an Open Access article distributed under the terms of the Creative Commons Attribution Non-Commercial License (http://creativecommons.org/licenses/by-nc/4.0/) which permits unrestricted non-commercial use, distribution, and reproduction in any medium, provided the original work is properly cited. 
thod [1,2]. Furthermore, our previous papers including Kim et al. and Jung et al. reported that a lateral paracarotid out-ofplane approach (Song's technique) for real-time ultrasoundguided SGB using a standard high-frequency linear probe is a safe and suitable method [3,4]. However, the classical blind technique of SGB performed at C6 is less effective for sympathetic blockade of the upper extremity [5,6]. We speculated that a lateral paracarotid approach for ultrasound-guided SGB at the level of C6 may be also less effective in the upper extremity. Therefore, we attempted an ultrasound-guided SGB at the level of the 7th cervical vertebra (C7). This study aimed to compare the effect of the lateral paracarotid approach using ultrasound-guided SGB at C6 and C7 for sympathetic blockade in the upper extremity.

\section{MATERIALS AND METHODS}

\section{Patients}

After obtaining informed consent, 30 patients with sensorineural hearing loss or facial palsy were selected during the year of 2009, and referred to the pain clinic for SGB. Demographic information, including age, ratio of men to women, height and weight, is summarized in Table 1. All patients were American Society of Anesthesiologists physical status 1 or 2 , and did not have a problem with coagulation or a medication history of anticoagulants. All procedures were performed by a single physician for technical consistency. To reduce patient bias, each patient underwent repeat SGB twice - once at C6 and once at C7 - with a 3- to 5-day interval between blocks. The ultrasound device used in this study was equipped with a 6-13 MHz linear array probe (HFL38x, Sonosite M-turbo, Sonosite, Bothell, WA, USA).

After patients arrived at the pain clinic, a skin temperature indicator (MU-832RK, Life Scope 9, Nihon Koden, Japan)

Table 1. Demographic data

\begin{tabular}{lc}
\hline Number of patients $(\mathrm{M} / \mathrm{F})$ & $30(11 / 19)$ \\
Age $(\mathrm{yr})$ & $52 \pm 18.3(19-89)$ \\
Weight $(\mathrm{kg})$ & $60.3 \pm 9.16(48-84)$ \\
Height $(\mathrm{cm})$ & $160.4 \pm 9.32(141-176)$ \\
BMI $\left(\mathrm{kg} / \mathrm{m}^{2}\right)$ & $23.4 \pm 2.6(20.0-25.1)$ \\
\hline
\end{tabular}

Values are presented as number of patients or mean \pm standard deviation (range).

BMI, body mass index. was placed onto the hypothenar area of the ipsilateral hand. After a 5-min period to allow for temperature stabilization, SGB was performed. Because skin temperature can be influenced by the surrounding environment, room temperature was maintained at $22-25^{\circ} \mathrm{C}$.

Patients were positioned supine, and a $10 \mathrm{~cm}$ pillow was placed beneath the shoulders to aid in neck extension. For SGB at C6, a lateral paracarotid out-of-plane approach was performed for ultrasound-guided SGB (Fig. 1) [3,4]. A total of $4 \mathrm{~mL}$ of $0.2 \%$ ropivacaine was injected to achieve blockade. For SGB at C7, a lateral paracarotid approach was also used. First, however, the ipsilateral paratracheal transverse view of the neck showing Chassaignac's tubercle at $\mathrm{C} 6$ was confirmed under the guidance of a standard high-frequency linear probe. In this short-axis view, the prominent anterior tubercle of the C6, the 6th cervical nerve root, and the oval shape structure of the longus colli muscle were revealed. The probe was then moved caudally to $\mathrm{C}$, which was confirmed by the $\mathrm{C} 7$ transverse process with a single posterior tubercle. In addition, the target of $\mathrm{C} 7$ was determined by the vertebral artery using the color Doppler mode, which came up to the tubercle of the cervical spine. At the level of $\mathrm{C}$, the vertebral artery lies at the anterior $\mathrm{C} 7$ transverse process in an extraforaminal location and originates from the subclavian artery at the inferior border of the thyroid gland. After confirming blood vessels using the color Doppler mode, a 25-gauge, 4 $\mathrm{cm}$ needle (Kovac Med Inc, Ansan, Korea) was used to pene-

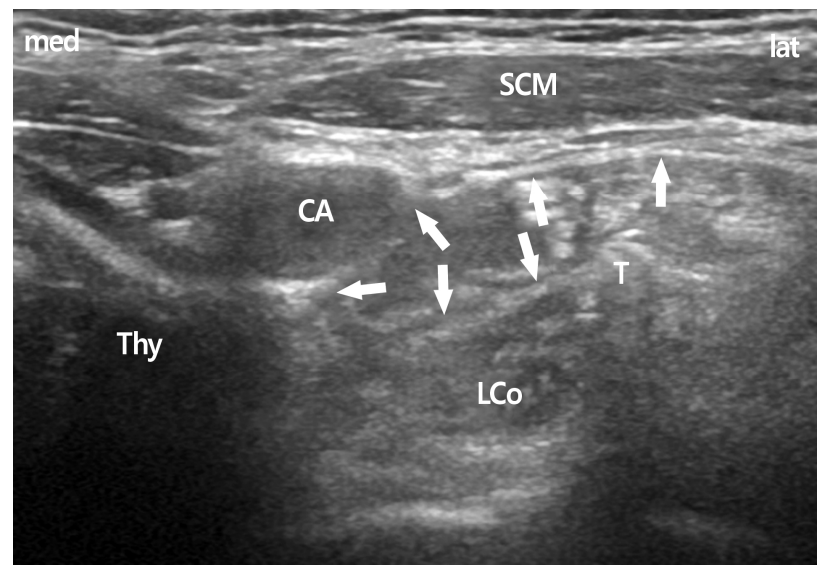

Fig. 1. The spread of local anesthetics at C6 level. Arrows show the subfacial spread of local anesthetics above the longus colli muscle. CA, carotid artery; LCo, longus colli muscle; SCM, sternocleidomastoid muscle; Thy, thyroid gland; T, Chassaignac's tubercle; med, medial; lat, lateral. 
trate the compressed internal jugular vein using the out-ofplane approach and aimed 15 degrees postero-medially toward the longus colli muscle beneath the prevertebral fascia. The exact target of this block technique is the space between the prevertebral fascia and the longus colli muscle. After reaching the target, intravascular placement of the needle was ruled out with a negative aspiration test, followed by injection of $0.2-0.3 \mathrm{~mL}$ of local anesthetic. During the initial injection of a small dose, correct needle-tip placement was confirmed by the spreading of local anesthetic above the longus colli beneath the prevertebral fascia. Additional injections were divided into amounts $<1 \mathrm{~mL}$; the needle was repositioned several times to maintain its exact position and to avoid supra-prevertebral fascial injection. Finally, a spreading hypoechoic lake of local anesthetic was observed circumferentially around the longus colli. A total of $4 \mathrm{~mL}$ of $0.2 \%$ ropivacaine was injected to achieve blockade (Fig. 2).

The injection site was compressed manually for $5 \mathrm{~min}$ to avoid the formation of hematoma due to penetration of the internal jugular vein. The effect of SGB was confirmed by the onset of Horner's sign. The temperature of the hypothenar area was recorded at 1, 2, 3, 4, 5, 8, 10, and $15 \mathrm{~min}$. Side effects, such as hoarseness, hematoma and upper extremity weakness, were also verified by the physician during the patient's hospital stay.

After collecting all data, the largest increases in skin tem-

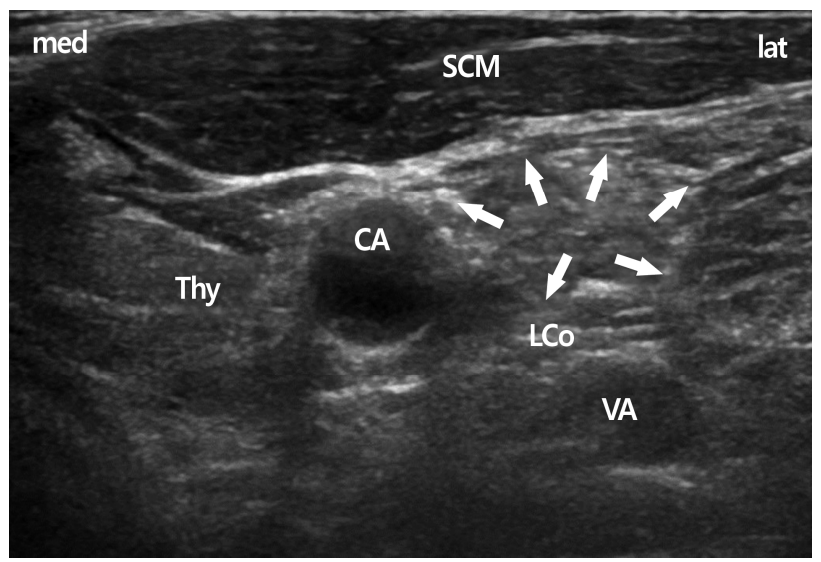

Fig. 2. In the C7 transverse view, the vertebral artery come out from the tubercle and is seen easily. Arrows show the subfascial spread of local anesthetics above the longus colli muscle. CA, carotid artery; LCo, longus colli muscle; SCM, sternocleidomastoid muscle; Thy, thyroid gland; VA, vertebral artery; med, medial; lat, lateral. perature after SGB (post-SGB) were compared with skin temperatures before SGB (pre-SGB) in each patient. The difference between pre-SGB and post-SGB temperatures were compared between SGBs performed at C6 and C7. Additionally, the number of patients who exhibited significant elevation of post-SGB temperature $\left(>1{ }^{\circ} \mathrm{C}\right)$ compared with preSGB were compared between SGBs performed at $\mathrm{C} 6$ and $\mathrm{C} 7$.

\section{Statistical analysis}

All data were analyzed using SPSS version 15.0 for Windows (SPSS Inc., Chicago, IL, USA). The paired samples t-test was used to compare temperature increases between SGB performed at C6 and C7. The Student's t-test, chi-squared, and Fisher's exact tests were used to compare differences in increased temperature, the number of patients with significant increase in post-SGB temperature, and side effects; $p<0.05$ was considered to be statistically significant.

\section{RESULTS}

Each patient underwent SGB separately: once at C6 and once at C7. After SGB, all patients exhibited cranial sympathetic blockade symptoms. In patients who underwent ultrasound-guided SGB at C6 using the lateral paracarotid approach, the mean pre-SGB temperature was $35.0 \pm 1.08^{\circ} \mathrm{C}$ and post-SGB was $35.5 \pm 1.09^{\circ} \mathrm{C}$, with a difference that was statistically significant $(p<0.05)$. However, the mean difference between post-SGB and pre-SGB temperature was $0.50 \pm 0.38$ ${ }^{\circ} \mathrm{C}$ (range, $0-1.3^{\circ} \mathrm{C}$ ).

In patients who underwent ultrasound-guided SGB at C7 using the lateral paracarotid approach, the mean pre-SGB temperature was $34.2 \pm 1.59^{\circ} \mathrm{C}$ and post-SGB was $35.6 \pm 1.07$ ${ }^{\circ} \mathrm{C}$, a difference that was statistically significant $(p<0.05)$. The difference between post-SGB and pre-SGB temperatures was $1.41 \pm 0.68^{\circ} \mathrm{C}$ (range, $0.1-3.1^{\circ} \mathrm{C}$ ).

There were statistically significant differences between preSGB and post-SGB temperatures with blocks performed at C6 and C7 ( $p<0.05$ [Student's t-test]) (Table 2). Significantly increased post-SGB temperatures $\left(>1{ }^{\circ} \mathrm{C}\right)$ were recorded in $5 / 30(16.7 \%)$ and $24 / 30(80 \%)$ for SGBs performed at C6 and C7, respectively ( $p<0.05$ [Chi-Square test]) (Table 3). The number of effective sympathetic blocks was higher when per- 
Table 2. Comparison of pre-SGB and post-SGB temperatures in C6 and C7 groups $(n=30)$

\begin{tabular}{llc}
\hline Temperature & C6 & C7 \\
\hline Pre-SGB $\left({ }^{\circ} \mathrm{C}\right)$ & $35.0 \pm 1.08$ & $34.2 \pm 1.59$ \\
Post-SGB $\left({ }^{\circ} \mathrm{C}\right)$ & $35.5 \pm 1.09^{\mathrm{a})}$ & $35.6 \pm 1.07^{\mathrm{a})}$ \\
The differences between post- and pre-SGB $\left({ }^{\circ} \mathrm{C}\right)\left(\right.$ range, $\left.{ }^{\circ} \mathrm{C}\right)$ & $0.50 \pm 0.38(0.0-1.3)$ & $1.41 \pm 0.68^{\mathrm{b})}(0.1-3.1)$ \\
\hline
\end{tabular}

Values are presented as mean \pm standard deviation.

SGB, stellate ganglion block; C6, 6th cervical vertebra; C7, 6th cervical vertebra.

a) $p<0.05$, there is significant difference between pre-SGB and the most increased post-SGB temperatures in each group ( $p<0.05$, student t-test). ${ }^{\text {b) }} p<0.05$, compared with C6 group.

Table 3. The number of patients revealed significantly increased temperature $\left(>1^{\circ} \mathrm{C}\right)$ after SGB in C6 and C7 groups ( $\mathrm{n}=30$ )

\begin{tabular}{lcc}
\hline Number of patients & C6 (\%) & C7 (\%) \\
Increased temperature more than $1{ }^{\circ} \mathrm{C}$ & $5(16.7)$ & $24(80)^{\mathrm{a})}$ \\
Increased temperature less than $1{ }^{\circ} \mathrm{C}$ & $25(83.3)$ & $6(20)$
\end{tabular}

SGB, stellate ganglion block; C6, 6th cervical vertebra; C7, 6th cervical vertebra.

a) $p<0.05$, there is statistical differences between $\mathrm{C} 6$ and $\mathrm{C} 7$ group (Chi-Square test). There are more significant patients revealed effective sympathetic block in group C7 than in group C6.

formed at C7 compared with C6.

Regarding side effects after SGB, the incidence of hoarseness was $4(13.3 \%)$ and $2(6.7 \%)$ in blocks performed at C6 and C7, respectively, with no occurrences of hematoma. Upper extremity weakness occurred in 2 (6.7\%) and 1 (3.3\%) patients who underwent SGB at C6 and C7, respectively. There were no significant differences in hoarseness or upper extremity weakness $(p>0.05)$ (Table 4$)$.

\section{DISCUSSION}

The sympathetic ganglia of the cervical spine consist of the superior, middle, inferior, and cervicothoracic ganglions. Commonly, the 7th cervical sympathetic ganglion and the 1st thoracic sympathetic ganglion are fused into the cervicothoracic ganglion, and called the "stellate ganglion". It is located immediately anterior to the transverse process of the C7 vertebra, and superior or anterior to the neck of the first rib [7]. This ganglion is described as a structure 1 to $2.5 \mathrm{~cm}$ long, approximately $1 \mathrm{~cm}$ wide and $0.5 \mathrm{~cm}$ thick. SGBs are used to block sympathetic innervations of cranial, cervical, and upper thoracic lesions [7,8]. It is a common intervention in the diagnosis and management of sympathetically mediated pain and vascular insufficiency of the face and upper extremity. In addition, this sympathetic block has been advocated for treatment of a variety of medical conditions including phantom pain, postherpetic neuralgia, cancer pain, car-
Table 4. Side effects following SGB in C6 and C7 groups $(n=30)$

\begin{tabular}{lll}
\hline Number of patients & \multicolumn{1}{c}{ C6 } & \multicolumn{1}{c}{ C7 } \\
\hline Hoarseness (\%) & $4(13.3)$ & $2(6.7)$ \\
Arterial puncture (\%) & $0(0)$ & $0(0)$ \\
Hematoma (\%) & $0(0)$ & $0(0)$ \\
Upper extremity weakness (\%) & $1(3.3)$ & $2(6.7)$ \\
\hline
\end{tabular}

There were no significant difference between two groups $(p>$ 0.05 , Fisher's exact test).

SGB, stellate ganglion block; C6, 6th cervical vertebra; C7, 6th cervical vertebra.

diac arrhythmia, orofacial pain, vascular headache, and complex regional pain syndrome.

Although the location or presence of the stellate ganglion varies among patients, SGB at C6 is commonly performed using a landmark technique owing to the easy palpability of Chassaignac's tubercle. However, investigators using magnetic resonance imaging reported that $10 \mathrm{~mL}$ of normal saline blindly injected at the level of C6 would not likely reach the stellate ganglion. Consequently, it results in failure of sympathetic block of upper extremity lesions because of technical failure during injection or inadequate spread of local anesthetics to the stellate ganglion [9]. Previously, we also attempted to determine an optimal volume for the blind landmark technique for SGB at C6 on appropriate sympathetic blockade of the ipsilateral upper extremity by measuring the elevation of skin temperature [6. The authors reported that effective SGB at C6 on the upper extremity required larger vol- 
umes of local anesthetic $(>12 \mathrm{~mL})$ and recommended lower doses for SGB at C7 or T1. These results suggest that successful SGB at C6 using a blind landmark technique requires a larger volume of drug to treat upper extremity pathology.

Hogan et al. performed computed tomography-guided local anesthetic injection at the level of T1 [10], and reported that injection at T1 was more effective than paratracheal injection at $\mathrm{C} 6$ in achieving effective sympathetic block of the upper extremity. Smith reported successful SGB at C7 in $>400$ patients [11]. Moore and Bridenbaugh also successfully performed SGB at C7 without a serious complication in $>2,000$ blocks [12].

Nevertheless, SGB at C7 may be associated with more risk than SGB at C6. It may cause severe complications such as injury to the vertebral artery, inadvertent intra-arterial injection of local anesthetic, and pneumothorax [13-15]. If the vertebral artery is punctured inadvertently, it is impossible to stop bleeding by compression; consequently, surgery would be required. The accidental intra-arterial injection of local anesthetics may cause central nervous system toxicity, coma, and/or fulminant tonic clonic seizure. The vertebral artery arises from the subclavian artery, and then enters deep to the transverse process at the level of the C7 [16]. Furthermore, the thoracic cage is near, and the risk for pneumothorax is elevated.

Despite the above-mentioned risks, SGB at C7 is feasible and safe using ultrasound, without the risk for injury to arteries and other important structures. Ultrasound-guided SGB has several advantages, including safety, rapid onset of Horner's sign and lower volumes of local anesthetic, compared with the landmark technique $[1,4,17]$. Authors have already reported that the lateral paracarotid out-of-plane approach (Song's technique) for real-time ultrasound-guided SGB at C6 is a safe and suitable method [3,4]. Lee et al. reported that a $2 \mathrm{~mL}$ dose was sufficient for successful SGB when performing ultrasound-guided SGB at C6 [18]. However, SGB at C6 remains a concern because of the weak effect of sympathetic blockade in the ipsilateral upper extremity. Therefore, we attempted to determine an easier way to safely succeed with SGB at C7 using ultrasound guidance. In addition, this study also compare the effectiveness of the lateral paracarotid approach for ultrasound-guided SGB at C6 and C7 in increasing skin temperature in the ipsilateral upper extremity. The results would indicate the level at which SGB would be more effective in upper extremity pathologies including Raynaud's syndrome and complex regional pain syn drome.

After SGB, the temperature of the upper extremity increased to some extent according to the level of SGB. At C6, the mean increase in temperature was $0.50 \pm 0.38^{\circ} \mathrm{C}$, and only $5(16.7 \%)$ patients exhibited significantly increased $\left(>1{ }^{\circ} \mathrm{C}\right)$ post-SGB temperatures. These data suggest that SGB at C6 may be insufficient to achieve successful sympathetic blockade in the upper extremity. When SGB performed at C7, the mean increase in temperature was $1.41 \pm 0.68{ }^{\circ} \mathrm{C}$, and 24 $(80 \%)$ patients exhibited significantly increased $\left(>1{ }^{\circ} \mathrm{C}\right)$ temperatures after SGB. The number of effective sympathetic blocks was significantly higher when performed at $\mathrm{C} 7$ than at C6.

Although Lee et al. reported that a $2 \mathrm{~mL}$ dose was sufficient for spread below $\mathrm{C} 7$ and $\mathrm{T} 1$ junction when performing ultrasound-guided SGB at C6 [18], the conventional injection technique using a $4 \mathrm{~mL}$ dose at $\mathrm{C} 6$ in our clinics has demonstrated less efficient sympathetic block in the upper extremity than at C7. In the course of needle repositioning between the prevertebral fascia and longus colli muscle, local anesthetics were probably administered to the longus colli muscle. Consequently, this may result in insufficient spread below C7 in SGB performed at C6.

While performing SGB at C7, however, there are many considerations to be mindful of. The esophagus is more lateral to the trachea at the level of C7. The incidence of the major vessels, including arteries that appear in long axis view, such as the inferior thyroidal artery, was higher than that at C6. Therefore, an ultrasound-guided approach at C7 may be a less safe approach than that at $\mathrm{C} 6$ because there are several important anatomical structures including the esophagus and blood vessels [19]. Nevertheless, we chose the lateral paracarotid approach with ultrasound-guided SGB at C7 for sympathetic block in the upper extremity. This approach permitted a shorter length of needle pathway than the lateral approach using in-plane needle insertion, and the requirement for lower volume of drug than the lateral paracarotid approach with ultrasound-guided SGB at C6. In addition, ultrasound enables physicians to visualize the esophagus and blood vessels.

According to these results, we recommend a lateral paracarotid approach for ultrasound-guided SGB at C7, which 
can increase the effectiveness of sympathetic block more than SGB performed at C6 to treat upper extremity pathologies. This approach method for ultrasound-guided SGB at C7 yields better results for sympathetic blockade of the upper extremity, with no more complications than at C6. Moreover, it is within the range of possibility for all practitioners to easily perform SGB at C7. Therefore, we suggest that a lateral paracarotid out-of-plane approach for ultrasound-guided SGB at C7 could be a useful method for treating sympathetic-mediated disease in the upper extremity.

\section{CONFLICT OF INTEREST}

No potential conflict of interest relevant to this article was reported.

\section{ORCID}

Jongyoon Baek, https://orcid.org/0000-0003-4007-770X

Bum Soo Kim, https://orcid.org/0000-0001-5604-8554

Hwarim Yu, https://orcid.org/0000-0002-4020-1793

Hyuckgoo Kim, https://orcid.org/0000-0002-3879-6441

Chaeseok Lim, https://orcid.org/0000-0002-6596-2601

Sun Ok Song, https://orcid.org/0000-0003-2236-4422

\section{REFERENCES}

1. Narouze S, Vydyanathan A, Patel N. Ultrasound-guided stellate ganglion block successfully prevented esophageal puncture. Pain Physician 2007;10:747-52.

2. Narouze S. Ultrasound-guided stellate ganglion block: safety and efficacy. Curr Pain Headache Rep 2014;18:424.

3. Kim H, Song SO, Jung G. A lateral paracarotid approach for ultrasound-guided stellate ganglion block with a linear probe. J Anesth 2017;31:458-62.

4. Jung G, Kim BS, Shin KB, Park KB, Kim SY, Song SO. The optimal volume of $0.2 \%$ ropivacaine required for an ultrasound-guided stellate ganglion block. Korean J Anesthesiol
2011;60:179-84.

5. Moore DC. Therapeutic stellate ganglion block: 5 versus 10 $\mathrm{mL}$ of a local anesthetic. Reg Anesth Pain Med 2008;33:191-2.

6. Song SO, Jo YW. Effects of the volume of local anesthetic used in stellate ganglion block on the elevation of skin temperature of ipsilateral upper extremity. Korean J Anesthesiol 1999;37:233-9. Korean.

7. Elias M. Cervical sympathetic and stellate ganglion blocks. Pain Physician 2000;3:294-304.

8. Hogan QH, Erickson SJ. MR imaging of the stellate ganglion: normal appearance. AJR Am J Roentgenol 1992;158:655-9.

9. Hogan QH, Erickson SJ, Haddox JD, Abram SE. The spread of solutions during stellate ganglion block. Reg Anesth 1992; 17:78-83.

10. Hogan QH, Erickson SJ, Abram SE. Computerized tomography-guided stellate ganglion blockade. Anesthesiology 1992; 77596-9.

11. Smith DW. Stellate ganglion block; the tissue displacement method. Am J Surg 1951;82:344-8.

12. Moore DC, Bridenbaugh LD Jr. The anterior approach to the stellate ganglion use without a serious complication in two thousand blocks. J Am Med Assoc 1956;160:158-62.

13. Higa K, Hirata K, Hirota K, Nitahara K, Shono S. Retropharyngeal hematoma after stellate ganglion block: Analysis of 27 patients reported in the literature. Anesthesiology 2006; 105:1238-45

14. Moore DC. An unusual complication after stellate ganglion block. Br J Anaesth 1964;36:601.

15. Uchida T, Nakao S, Morimoto M, Iwamoto T. Serious cervical hematoma after stellate ganglion block. J Anesth 2015; 29:321.

16. Hong JT, Park DK, Lee MJ, Kim SW, An HS. Anatomical variations of the vertebral artery segment in the lower cervical spine: analysis by three-dimensional computed tomography angiography. Spine (Phila Pa 1976) 2008;33:2422-6.

17. Kapral S, Krafft P, Gosch M, Fleischmann D, Weinstabl C. Ultrasound imaging for stellate ganglion block: direct visualization of puncture site and local anesthetic spread. A pilot study. Reg Anesth 1995;20:323-8.

18. Lee MH, Kim KY, Song JH, Jung HJ, Lim HK, Lee DI, et al. Minimal volume of local anesthetic required for an ultrasound-guided SGB. Pain Med 2012;13:1381-8.

19. Bhatia A, Flamer D, Peng PW. Evaluation of sonoanatomy relevant to performing stellate ganglion blocks using anterior and lateral simulated approaches: an observational study. Can J Anaesth 2012;59:1040-7. 